\title{
Organic electrochemical transistors for signal amplification in fast scan cyclic voltammetry
}

\author{
Klas Tybrandt, Suresh Babu Kollipara and Magnus Berggren
}

\section{Linköping University Post Print}

\section{Tweet}

N.B.: When citing this work, cite the original article.

Original Publication:

Klas Tybrandt, Suresh Babu Kollipara and Magnus Berggren, Organic electrochemical transistors for signal amplification in fast scan cyclic voltammetry, 2014, Sensors and actuators. B, Chemical, (195), 651-656.

http://dx.doi.org/10.1016/j.snb.2014.01.097

Copyright: Elsevier

http://www.elsevier.com/

Postprint available at: Linköping University Electronic Press

http://urn.kb.se/resolve?urn=urn:nbn:se:liu:diva-105894 


\title{
Organic electrochemical transistors for signal amplification in fast scan cyclic voltammetry
}

\author{
Klas Tybrandt ${ }^{\mathrm{a}}$, Suresh Babu Kollipara ${ }^{\mathrm{a}}$, Magnus Berggren ${ }^{\mathrm{a},{ }^{*}}$ \\ aLaboratory of Organic Electronics, Department of Science and Technology, Linköping University, 60174 \\ Norrköping, Sweden \\ *Corresponding author: Magnus Berggren, ITN, Linköpings Universitet, SE-601 74 Norrköping, Sweden. \\ Tel.: +46709783430 \\ E-mail address: magnus.berggren@liu.se
}

\begin{abstract}
Fast scan cyclic voltammetry (FSCV) is an electrochemical method commonly used in neuroscience for spatiotemporal measurement of the concentration of dopamine and other electroactive species. Since FSCV involves wide bandwidth measurements of low currents, the technique is normally very sensitive to electrical noise and is typically performed inside a Faraday cage. In order to reduce the electrical noise and to enable measurements in an unshielded environment, we take use of an organic electrochemical transistor (OECT) to amplify the FSCV signals. OECTs based on the conducting polymer poly(3,4-ethylenedioxythiophene):poly(styrenesulfonate) (PEDOT:PSS) were microfabricated and characterized. A patterned $10 \mu \mathrm{m}$ gold microelectrode was used as the sensing electrode and the FSCV signal was amplified by the OECT. With this approach, successful measurements of dopamine concentrations in the $10 \mu \mathrm{M}$ range were performed in a completely unshielded measurement setup. Our results demonstrate how OECTs can successfully be used, in an on-site amplification application to characterize biochemical signals, thus open up new trails for flexible multifunctional organic bioelectronics systems.
\end{abstract}

\section{Keywords}

organic electrochemical transistor, fast scan cyclic voltammetry, bioelectronics, dopamine, onsite amplification 


\section{Introduction}

The detection and quantification of neurochemical species in vitro and in vivo is of great importance within neuroscience. Such measurements have made a large contribution to our understanding of the signaling cascades of the brain and its associated diseases.[1] Further, real time measurements of neurochemical species in the brain may open up possibilities to detect and prevent emerging seizures and other brain disorders.[2] Electrochemical methods combine the advantages of high spatiotemporal resolution and purely electronic instrumentation, which promise for easy implantation. Several electrochemical methods are used within neuroscience, which relay on the oxidation and reduction of electrochemically active neurochemical species at sensing electrodes. The methods primarily differ in the sense how the potential is applied and how the resulting signal is transduced and interpreted. Fast scan cyclic voltammetry (FSCV) is a method of special interest as it combines spatiotemporal resolution with a relatively high chemical selectivity.[3] A triangular waveform is applied to the sensing electrode, often represented by a carbon fiber electrode, with a periodicity of $100 \mathrm{~ms}$. Typically, the resting potential is found in the range of $[-0.4,0] \mathrm{V}(\mathrm{vs} \mathrm{Ag} / \mathrm{AgCl})$ and the maximum potential applied is typically in the range of $[0.6,1.4] \mathrm{V}$. What distinguishes FSCV from ordinary CV is the relatively high scan speed, often ranging from 50 to $400 \mathrm{~V} / \mathrm{s}$. The fast scan speed generates a large nonfaradic contribution to the current, which is subtracted from the voltammogram in order to isolate the faradic current, i.e. to isolate the current originating from the specific oxidation/reduction reaction of interest. Thus FSCV is a differential method in the sense that differences between subsequent scans recorded at various conditions are analyzed.[1] To estimate the concentration of a specific species, a background scan is first performed in absence of the species of interest. Then, the resulting backgroundsubtracted voltammogram is achieved, which provide information about the actual concentration of the species. The chemical selectivity of the method is a consequence of that many neurochemical species have different oxidation potentials and reaction kinetics, and can thus be distinguished in the voltammogram.[4] Further, the high scan speed favors species with fast reaction kinetics over slower ones, which allows for in vivo detection of the neurotransmitter dopamine, which is of great importance to many neurological processes. Thus, sub $\mu \mathrm{M}$ concentrations of dopamine can be detected in the brain by FSCV even in the presence of hundreds of $\mu \mathrm{M}$ of ascorbic acid.[5]

FSCV is very sensitive to electrical noise as it involves wide bandwidth measurements of low currents and is typically performed inside a Faraday cage. In order to reduce the uptake of electrical noise it is desirable to amplify the signal as close to the sensing site as possible. This can potentially improve the sensitivity and should also enable measurements in an unshielded environment, thereby 
opening up for new FSCV applications. Organic thin film transistors is a promising class of transistors for such on-site amplifications.[6, 7] They have recently received significant attention for biosensing applications due to their potential for low cost manufacturing, flexibility, unconventional geometries, biocompatibility and high sensitivity.[8-11] Organic electrochemical transistors (OECTs) based on the conducting polymer poly(3,4-ethylenedioxythiophene):poly(styrenesulfonate) (PEDOT:PSS) seems especially attractive for certain bioapplications as PEDOT:PSS is stable in physiological electrolytes, have high electronic and ionic conductivity and have been proven biocompatible.[12, 13] The PSS phase facilitates ionic conduction in the material and thereby enables modulation of the oxidation state throughout the entire bulk of the PEDOT:PSS material. A change in oxidation state of PEDOT affect its electrical conductivity of the material system, which can be used as a sensor signal transduction functionality.[14] A key aspect in most sensing applications based on OECTs is to optimize the gate capacitance in relation to the channel capacitance in order to achieve maximum sensitivity.[15] To date, PEDOT:PSS-based OECTs have been used in a plethora of applications, including enzymatic sensing[11], DNA detection[16], sensing of antibodies[17] and studies of cell layer formation[18]. Within neuroscience it has been shown that PEDOT:PSS coatings can improve the recording capabilities of neural electrodes and recently PEDOT:PSS-based OECTs were used to improve neuronal signal recordings by amplification of the signal on-site.[13, 19] Further, PEDOT:PSS electrodes have been used in amperometric detection of several electroactive neurochemical species.[20]

Inspired of the favorable properties of OECTs, here we report on the incorporation of an OECT in the close proximity of the sensing electrode for on-site amplification in a FSCV setup. The OECT has low impedance which allows for direct measurement with a standard DAQ card. The neurotransmitter dopamine is used to evaluate the sensing characteristics of the device. As microfabricated gold electrodes are used instead of the typical carbon fiber electrodes,[21] our employed approach allows for integration of additional components, which e.g. could be used to incorporate multiple sensing sites or complementary sensing technologies on the same devices. It should be noted, however, that carbon is preferred over gold for FSCV detection of dopamine due to electrochemical reasons.

\section{Materials and methods}

Titanium ( $5 \mathrm{~nm}$ ) and gold $(50 \mathrm{~nm})$ was thermally evaporated onto a 4 inch glass substrate. The electrodes were patterned with Shipley s1805 photoresist and sequentially wet etched. The photoresist was stripped in Shipley 1112A remover and the substrate was dry etched in a reactive ion etch (RIE) with 
an $\mathrm{O}_{2} / \mathrm{CF}_{4}$ plasma for $20 \mathrm{~s}$ to improve wetting. $0.5 \mathrm{v} / \mathrm{v} \%$ 3-glycidyloxypropyl)trimethoxysilane (Sigma) and $6 \mathrm{v} / \mathrm{v} \%$ ethylene glycol (Sigma) was added to a CLEVIOS ${ }^{\text {TM }}$ PH 1000 suspension (PEDOT:PSS). The solution was filtered through a $0.45 \mu \mathrm{m}$ PVDF filter and spin coated onto the substrate at $4000 \mathrm{rpm}$. The film was annealed for $30 \mathrm{~min}$ at $140^{\circ} \mathrm{C}$ and sequentially soaked in an ethylene glycol bath for $2 \mathrm{~min}$, washed and dried. A protective layer was spin coated at $3000 \mathrm{rpm}$ from a of poly(vinylidene fluoride-cohexafluoropropylene) containing solution ( $4 \mathrm{mg} / \mathrm{ml}$, in methyl ethyl ketone, Sigma). The layer was dried in a vacuum hot plate at $110^{\circ} \mathrm{C}$ for $90 \mathrm{~s}$ and a Shipley s1813 photoresist layer was patterned on top. The patterned was etched (RIE, $\mathrm{O}_{2} / \mathrm{CF}_{4}$ plasma, $30 \mathrm{~s}$ ) and stripped in acetone. After baking for $30 \mathrm{~min}$ at $110^{\circ}$ C, a $2 \mu \mathrm{m}$ thick layer of MicroChem SU-8 3000 was spin coated and patterned according to the instructions of the supplier. $\mathrm{Ag} / \mathrm{AgCl}$ paste (Advanced materials 500 ) was painted on the designated gate electrode and cured for $15 \mathrm{~min}$ at $110^{\circ} \mathrm{C}$. Finally, a ring was punched out of a PDMS disc and placed on top of the device prior to measurements. The full device is shown in Fig. 1.

A National Instruments DAQCard-6036E was controlled by a LabView program and used to supply the driving voltage and to measure $I_{D}$. A $3.26 \mathrm{k} \Omega$ resistor was connected in series with the drain terminal of the OECT and the voltage over the resistor was differentially measured at $100 \mathrm{kHz}$ with the DAQ card to obtain $I_{D}$. The source terminal was grounded and the driving voltage $V_{D}=-0.3 \mathrm{~V}$ was applied to the resistor. The gate voltage $V_{G}$ was supplied with an Agilent 33250A waveform generator which was connected to the gate terminal and ground. $\mathrm{A} 1 \mathrm{k} \Omega$ resistor was connected between the gate terminal and ground to supply the waveform generator with a constant output impedance and to reduce measurement noise. The reservoir was filled with $10 \mu$ I PBS buffer and the transfer curve (Fig. 2a) was obtained by linearly scanning $V_{G}$ between -0.3 and $0.4 \mathrm{~V}$ during 100 ms every 200 ms. PBS solutions containing $200 \mu \mathrm{M}$ ascorbic acid (Sigma) and varying dopamine concentration (0, 1.0, 2.6, 6.6, 16, 41, $256,640,1600$ and $4000 \mu M$, Sigma) was prepared prior to the measurement. Starting with the lowest dopamine concentration, $10 \mu$ of the analyte was added to the reservoir, measured, removed and replaced with a new analyte of higher concentration. The FSCV measurements were carried out by linearly scanning $V_{G}$ between -0.2 and $0.7 \mathrm{~V}$ at $50 \mathrm{~V} / \mathrm{s}$ every $100 \mathrm{~ms}$. The transient dopamine measurement was obtained by adding $1 \mu \mathrm{l}$ of $600 \mu \mathrm{M}$ dopamine solution during a measurement.

The collected data was analyzed with a MATLAB script. The measured voltage over the resistor was converted into the corresponding channel potential $\left(V_{C h}\right)$ of the OECT by using the transfer curve (Fig. 2a). The data was smoothed (Savitzky-Golay filter, 20 points) and a sine wave was fitted to the signal background and subtracted to remove the $50 \mathrm{~Hz}$ noise from the signal. The data was smoothed 
(Savitzky-Golay filter, 800 points), differentiated and multiplied with the effective capacitance from the CV in Fig. 2c and finally smoothed (Savitzky-Golay filter, 800 points) again to obtain $I_{G}$. During the FSCV measurement, the scanning potential applied to the gate $\left(V_{G}\right)$ is relative to the potential of the channel $\left(V_{C h}\right)$. In order to obtain the scanning potential versus $\mathrm{Ag} / \mathrm{AgCl}, V_{C h}$ was added to $V_{G}$. The background subtracted forward scan was calculated in the potential range for which both the background and measurement signal was available. A 5x5 Gaussian smoothing filter was applied to the color plot.

\section{Results and discussion}

The device structure comprising an OECT with the corresponding gate electrodes is shown in Fig. 1a. The gold electrodes are patterned on top of a glass substrate with the OECT channel ( $w=40 \mu \mathrm{m}, l=20 \mu \mathrm{m}, h$ $=60 \mathrm{~nm}$ ) patterned in between the source (S) and drain (D) terminals. A $2 \mu \mathrm{m}$ thick layer of SU-8 is patterned as an insulating layer with defined openings for the OECT channel, the gate electrodes and the contact pads. A ring of polydimethylsiloxane (PDMS) is glued on top of the device to serve as a reservoir for the analyte. The device comprises four circular sensing gate electrodes of different diameter $\left(d_{G 1}=5 \mu \mathrm{m}, d_{G 2}=10 \mu \mathrm{m}, d_{G 3}=20 \mu \mathrm{m}, d_{G 4}=40 \mu \mathrm{m}\right)$, intended to be used one at a time, in order to allow for optimal matching of the capacitance of the sensing gate electrode to the channel capacitance. Also, a large electrode covered with $\mathrm{Ag} / \mathrm{AgCl}$ paste is incorporated into the device for the characterization of the OECT. When scanning the potential of a sensing gate electrode, the generated current comprises a capacitive contribution from the changing of the electric double layer and a faradic contribution from the oxidation/reduction of dopamine. An electric current goes from the gate electrode, through the source and drain electrodes, to the OECT channel where it is compensated by an ionic current through the electrolyte of equal magnitude. This results in a modulation of the doping level (Fig. 1a) and thus the conductivity of the channel, which is electrically measured and constitutes the sensing signal.

The OECT was characterized in a phosphate buffered saline (PBS) solution with the $\mathrm{Ag} / \mathrm{AgCl}$ gate electrode. The source was grounded and the $V_{D}=-0.3 \mathrm{~V}$ was applied to a resistor in series with the drain terminal (Fig. 1a). The transfer curve, which relates the potential of the channel to the measured current, was obtained by measuring the drain current $I_{D}$ while scanning the gate voltage $V_{G}$ between -0.3 and $0.4 \mathrm{~V}$ with a periodicity of $100 \mathrm{~ms}$. The average of 20 consecutive forward scans is shown in Fig. 2 a. For low $V_{G}$ values the channel is heavily oxidized and the current through the OECT is high, while for low 
$V_{G}$ the channel is less oxidized and consequently the $I_{D}$ is relatively much lower. It is preferable to operate the transistor for $V_{G}=-0.1$ to $0.2 \mathrm{~V}$ since the transconductance $\left(g_{m}=\partial I D / \partial V\right)$ is largest in this region.

Another characteristic of interest is the evolution of $g_{m}$ with respect to the frequency of an applied oscillating voltage. $g_{m}$ is obtained by measuring $I_{D}$ while applying $V_{D}=-0.3 \mathrm{~V}$ and sweeping the frequency of a sinusoidal gate voltage of $0.1 \mathrm{~V}$ amplitude and $0 \mathrm{~V}$ dc bias (Fig. 2b). The $g_{m}$ is constant until $\sim 300 \mathrm{~Hz}$ where it starts to fall off and reaches $50 \%$ of its maximum value at around $1000 \mathrm{~Hz}$. This means that signal components above $300 \mathrm{~Hz}$ will be distorted while amplified by the transistor and thus the sensing gate in FSCV should be scanned with a significantly longer time period than $1 / 300 \approx 3.3 \mathrm{~ms}$ to allow for correct amplification of the sensing signal. The recorded $g_{m}$ starts to fall off at a lower frequency $(\sim 300 \mathrm{~Hz})$ than previously reported for an OECT $(\sim 1000 \mathrm{~Hz})$.[13] The reason for this may be that the channel length for the OECT studied here is $20 \mu \mathrm{m}$ as compared to $5 \mu \mathrm{m}$ for a previously reported device. Thus, it is expected that OECTs with a shorter channel length would enable faster scanning speeds in FSCV experiments.

In order to obtain the effective capacitance of the OECT channel, a CV measurement was performed on a $200 \mathrm{~nm}$ thick PEDOT:PSS film with an area of $28 \mathrm{~mm}^{2}$ (Fig. 2c). As indicated by the square-shaped CVs, the scan speed of $100 \mathrm{mV} / \mathrm{s}$ was concluded to be sufficiently low. The region of interest is marked in red, as this is the part of the CV spectrum that corresponds to the OECT operation in the FSCV experiment. The effective capacitance varies moderately in this region and it increases for lower potentials.

The ascorbic acid concentration in the brain lies between 200-400 $\mu \mathrm{M}$.[1] In order to mimic this in vivo environment, PBS solutions containing $200 \mu \mathrm{M}$ ascorbic acid were prepared. The dopamine concentrations were then varied and different solutions were evaluated in the OECT-amplified FSCV experiment. G2 $(\mathrm{d}=10 \mu \mathrm{m})$ was found to most favorably match the capacitance of the OECT channel and was therefore used throughout the experiment. The resting potential was set to $-0.2 \mathrm{~V}$ and a triangular waveform with the maximum potential of $0.7 \mathrm{~V}$ was scanned at $50 \mathrm{~V} / \mathrm{s}$. The gate current, i.e. the current from the sensing electrode, was estimated from the recorded drain current from:

$I_{G}=C\left(V_{C h}\left(I_{D}\right)\right) \cdot \frac{d V_{C h}\left(I_{D}\right)}{d t}$

where $V_{C h}\left(I_{D}\right)$ is the potential of the channel which was obtained from the transfer curve (Fig. 2b) and $C(V)$ is the capacitance of the channel which was obtained from the CV measurements performed on 
the PEDOT:PSS film (Fig. 2c). The average of 20 consecutive FSCV scans for different concentrations of dopamine is plotted in Fig. 3a. The peak at $\sim 0.5 \mathrm{~V}$ corresponds to the oxidation of dopamine while the reduction peak is visible at $\sim 0 \mathrm{~V}$. As can be seen in the $\mathrm{CV}$ there is a significant non-faradic contribution to the current. This capacitive contribution was then removed in the background-subtracted CV of the forward scan (Fig. 3b). Here, the oxidation peak at $\sim 0.5 \mathrm{~V}$ becomes even more evident and the amplitude $I_{O X}$ at a certain voltage can be used as a measure of the dopamine concentration. The peak values of the calculated currents are in good agreement with previously reported values of FSCV currents for gold electrodes.[21] The mean value and standard deviation of 20 consecutive $I_{O X}$ at $0.485 \mathrm{~V}$ are plotted versus the dopamine concentration in Fig. 3c. For low concentrations the sensitivity is high and the amplitude increases rapidly. For higher concentrations the sensitivity decreases as the response signal saturates. This behavior has previously been explained in terms of saturation of adsorbed dopamine to the sensing electrode.[21] The lower concentration range is of the greatest importance to many applications and is therefore plotted in Fig. 3d. For dopamine concentrations below $10 \mu \mathrm{M}$ the data points are rather scattered but for $c_{D A} \geq 16 \mu \mathrm{M}$ the data shows a nonlinear trend. The response is expected to be directly proportional to concentration for concentrations below $100 \mu \mathrm{M}$. Indeed, a line through the $16 \mu \mathrm{M}$ and $41 \mu \mathrm{M}$ data points passes through origin. This is consistent with the predicted response, however only two points is not enough to verify the direct proportionality in this experiment. It should be noted that the standard deviation in the measurement of each sample is $\sim 0.5 \mathrm{nA}$, which is significantly lower than the deviation between measurements of different samples. Thus, it is likely that the variation between different samples arises from the measurement procedure, in which the solution is removed and added to the well including the sensor.

To evaluate the interference of ascorbic acid to the dopamine detection, FSCV was performed on an analyte containing $400 \mu \mathrm{M}$ of ascorbic acid, thus twice the amount of the other analytes. The contribution to $I_{O X}$ at $0.485 \mathrm{~V}$ was found to be similar to that of $16 \mu \mathrm{M}$ dopamine (Fig. 4). Thus, small variations in the concentration of ascorbic acid has a limited effect on the accuracy of dopamine measurements, although the selectivity towards dopamine can be further improved by increasing the scan speed.[1] For that to be possible, however, the bandwidth of the transistor needs to be improved, as it preferably should be 10-40 times higher than the scan frequency of the CV. The low bandwidth of the employed OECT might be the reason for the smeared out shape of the CVs in Fig. 3a,b. This is a problem as it hampers the ability to distinct different electroactive species from each other in the CV. Thus, in order to perform faster CVs with less peak broadening, OECTs with a bandwidth of several kHz should be used. 
In most applications FSCV is used to detect transient concentration changes. To test how the OECT amplified FSCV responds in such a transient measurement, a drop of dopamine solution was added during measurement to a final concentration of $56 \mu \mathrm{M}$. The background-subtracted forward scans of the $10 \mathrm{~s}$ measurement are shown as color traces in Fig. $5 \mathrm{a}$. The addition of dopamine at $3 \mathrm{~s}$ can be seen as the oxidation peak emerges in the color map. The flat background and clear oxidation peak indicate that $56 \mu \mathrm{M}$ dopamine is well above the detection limit of the system. In Fig. $5 \mathrm{~b} \mathrm{I}_{O x}$ at $0.485 \mathrm{~V}$ have been converted into concentration by a linear fit from Fig. 3d. The increase of dopamine concentration at $\sim 3 \mathrm{~s}$ is clearly visible and the measured concentration increases over time as diffusion and convection transports the added dopamine to the sensing electrode. The standard deviation of the background noise is $\sim 1.5 \mu \mathrm{M}$, thus it is feasible to detect transient variations in dopamine concentration down to only a few $\mu \mathrm{M}$ with our OECT-based system.

When utilizing a transistor in direct contact with an electrolyte, on has to consider the implications of the applied voltage between the two terminals $\left(V_{D}\right)$. The applied voltage may induce electrolysis of reactive chemical species, which may affect the local biological environment and/or induce measurement errors. In previously reported OECT recordings of brain activity $V_{D}=-0.4 \mathrm{~V}$ was employed.[13] In this work $V_{D}$ was set to $-0.3 \mathrm{~V}$, however a lower voltage can be used if required by a biological setting. Electrolysis should not create significant measurement interference, as the current arising from electrolysis at a microelectrode is in the nA range while the current through the OECT is four orders of magnitude higher. Regardless, the area of bare metal contacts should be decreased in order to minimize electrolysis in in vitro and in vivo measurements.

\section{Conclusions}

For the first time, OECTs are reported for on-site amplification of FSCV signals. The on-site amplification allows for FSCV measurements in an unshielded environment, which opens up for new opportunities of FSCV in various sensor and monitoring applications. The performed transient measurement of dopamine concentration indicates that concentrations down to a few $\mu \mathrm{M}$ may be detected by our system. Since the dopamine concentration in the brain varies from several $\mu \mathrm{M}$ down to the $\mathrm{nM}$ range, the presented device should be able to measure some of the dopamine transients of physiological interest. However, in comparison to the current state of the art measurement equipment with Faraday cage, the reported system has inferior performance both with respect to detection limit and selectivity. Fortunately, this is the first study of OECTs for FSCV amplification and thus major improvements in sensitivity are to be 
expected. Gold may not be the ideal sensing electrode material and thus the addition of electrode coatings or surface treatments might improve the sensitivity of the device. The scan speed and thus the selectivity can be increased by reducing the channel length of the OECT. The OECT and sensing electrodes are microfabricated and may therefore be integrated with other components and devices on various substrates. This is especially appealing within the context of recent progress in neuronal recording, stimulation and biosensing with organic bioelectronics. By integrating several of these emerging technologies onto the same flexible substrates a new technology platform for signal transduction between the brain and electronics may be created.

\section{Acknowledgements}

The research was financed by VINNOVA (OBOE - Strategic Research Center for Organic Bioelectronics), the Knut and Alice Wallenberg Foundation, the Royal Swedish Academy of Science, the Swedish Research Council (621-2011-3517), Forum Scientium and the Önnesjö Foundation. 
Fig 1. Schematic illustration of the device. (a) The OECT terminals and the five gate electrodes consist of patterned gold on a glass substrate. The PEDOT:PSS OECT channel (blue) is patterned between the source (S) and drain (D) terminals. The electrodes are insulated by a SU-8 layer with openings for the channel and the sensing spots. A PDMS ring acts as a reservoir for the analyte. The sensing reaction of dopamine occurs at one of the sensing electrodes and the resulting ionic current is transferred through the electrolyte to the OECT channel where it modulates the doping level. When dopamine is oxidized cations are injected into the PEDOT:PSS channel which decreases the doping level and thus the conductivity of the channel. (b) Circuit diagram of the experimental setup utilized for the OECT-amplified FSCV measurements.

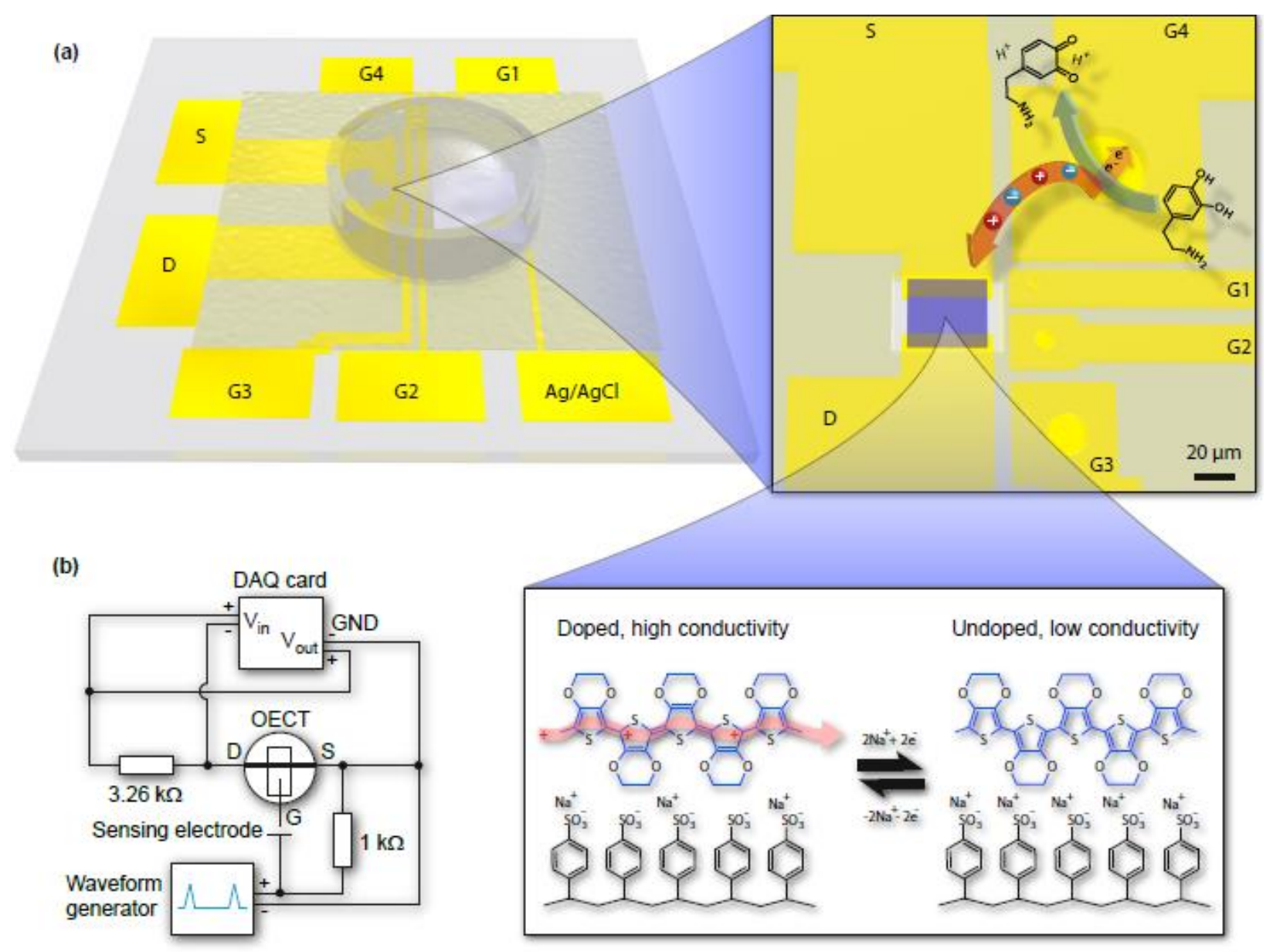


Fig. 2. OECT characteristics. (a) The transfer curve of the OECT for $V_{D}=-0.3 \mathrm{~V}$. (b) The frequency dependence of the transconductance of the OECT for $V_{D}=-0.3 \mathrm{~V}$ and $10 \mathrm{mV}$ gate amplitude. The transconductance starts to decrease for frequencies above $\sim 300 \mathrm{~Hz}$. (c) Cyclic voltammogram of a PEDOT:PSS film on top of a gold electrode in PBS buffer. The red part of the curve represents the region of interest for the FSCV experiment.

(a)

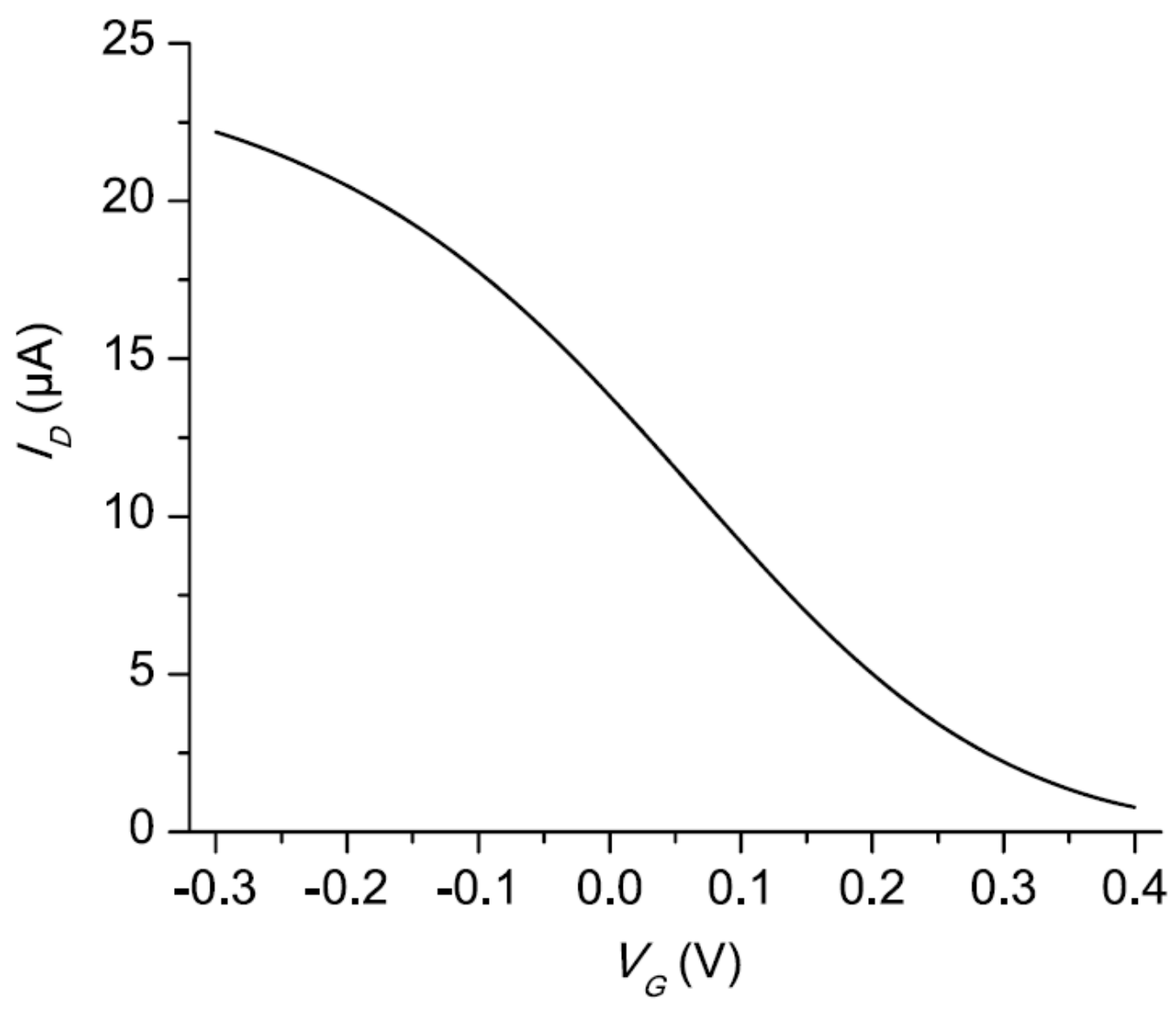


(b)

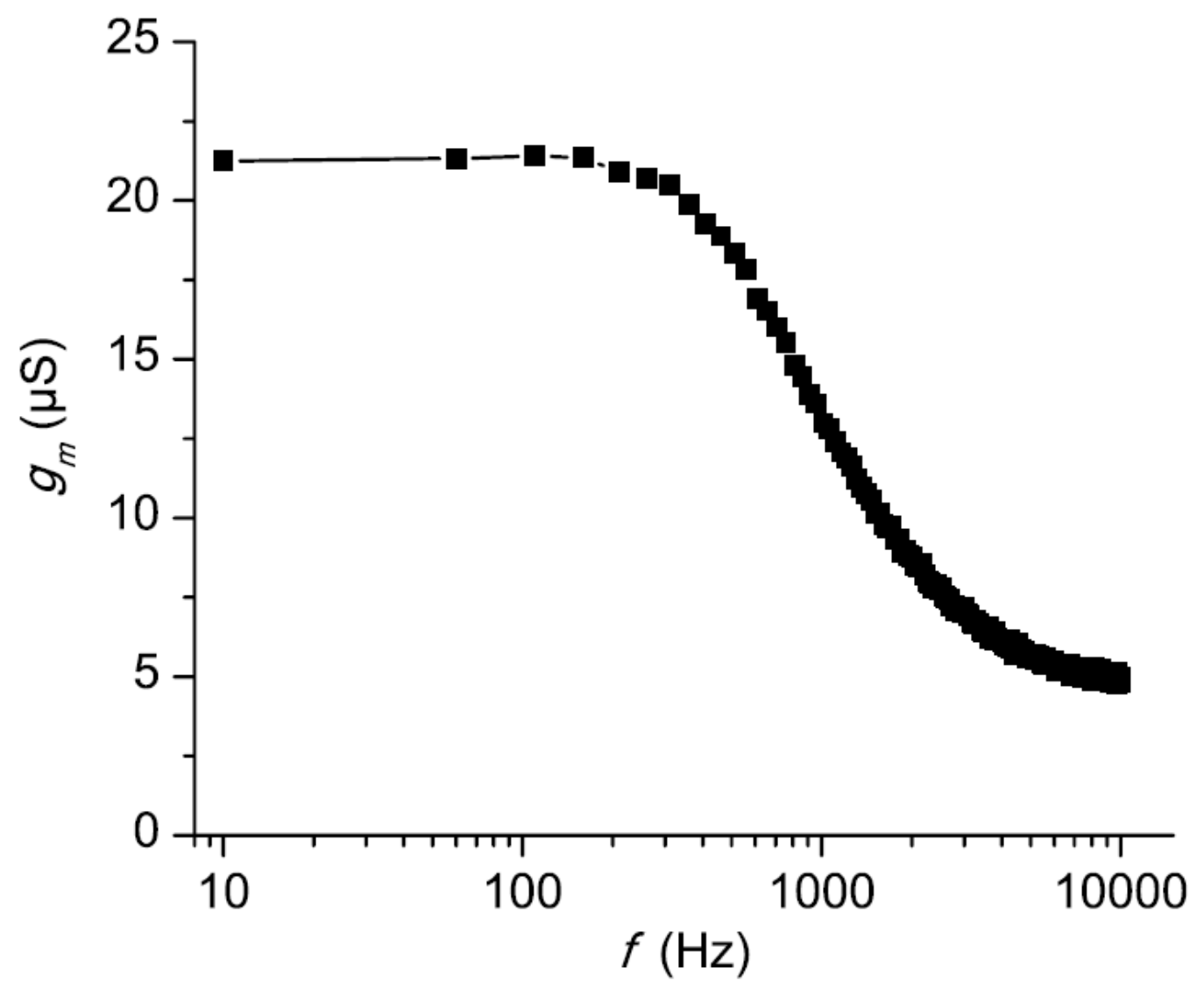


(c)

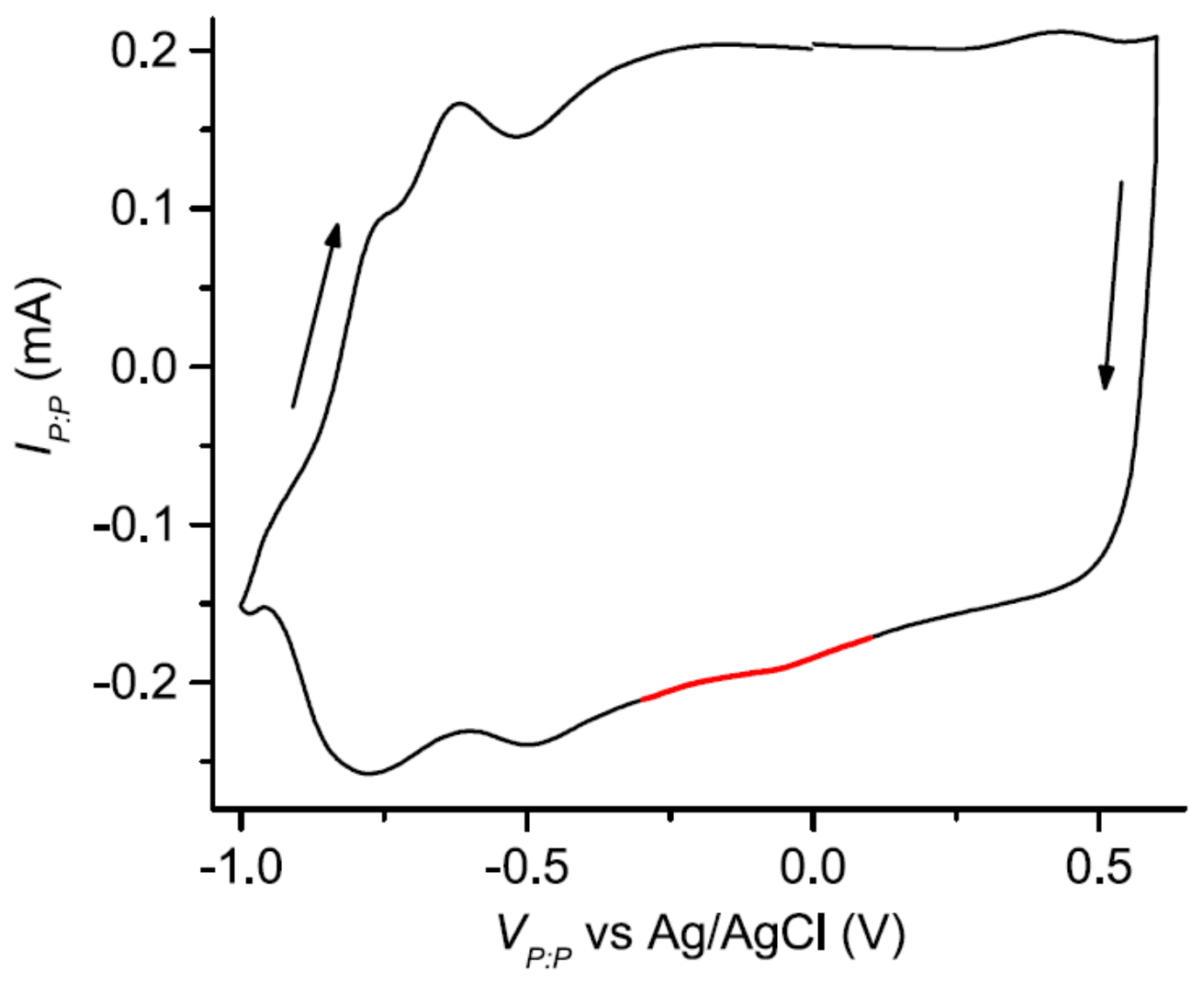


Fig. 3. Sensing of dopamine. (a) Cyclic voltammogram of FSCV measurements of analytes with varying dopamine concentration. The oxidation peak ( $0.5 \mathrm{~V})$ and the reduction peak ( $\sim \mathrm{V})$ are visible in the traces. (b) Background subtracted voltammogram (forward scans) of the same analytes as in (a). The amplitude of the oxidation peak can be used as a measure of the dopamine concentration. (c) Amplitude of the oxidation current at $0.485 \mathrm{~V}$ versus the dopamine concentration of the analyte. Mean value and standard deviations of 20 consecutive measurements are given. (d) Amplitude of the oxidation current at $0.485 \mathrm{~V}$ versus the dopamine concentration of the analyte for the lower concentration range of dopamine.

(a)

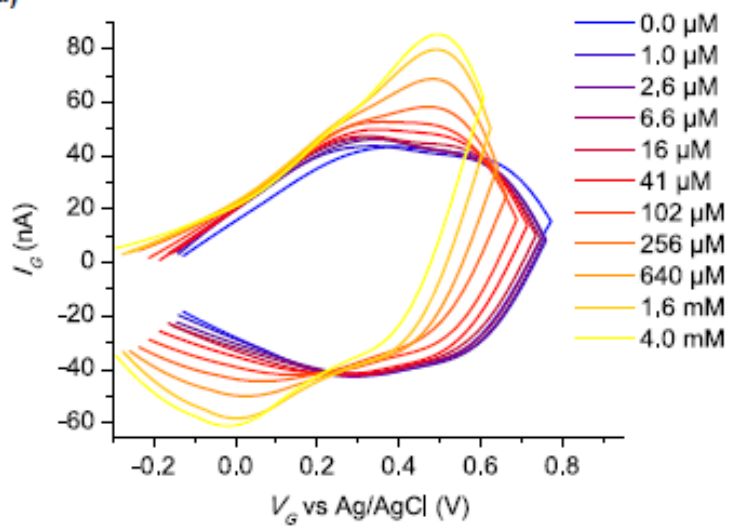

(c)

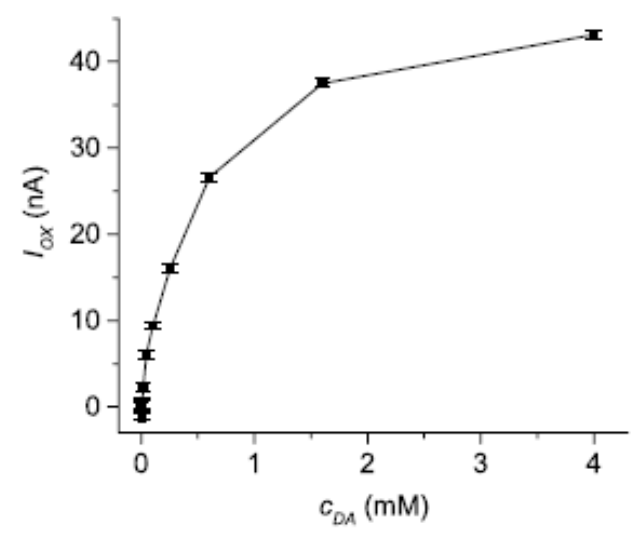

(b)

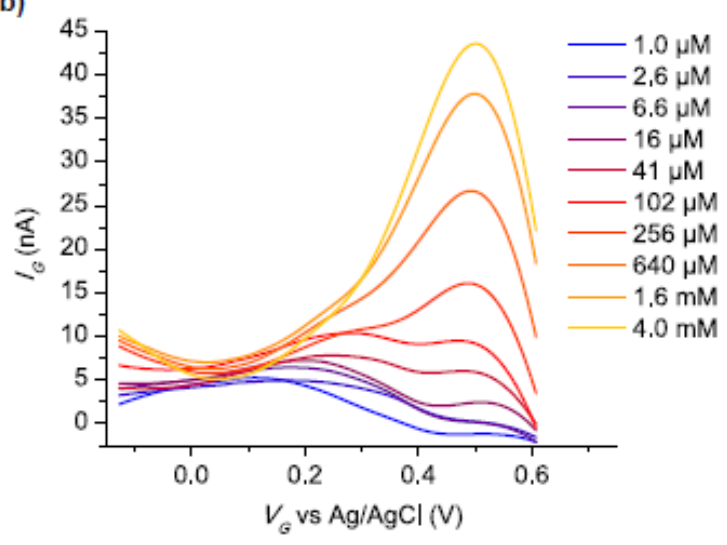

(d)

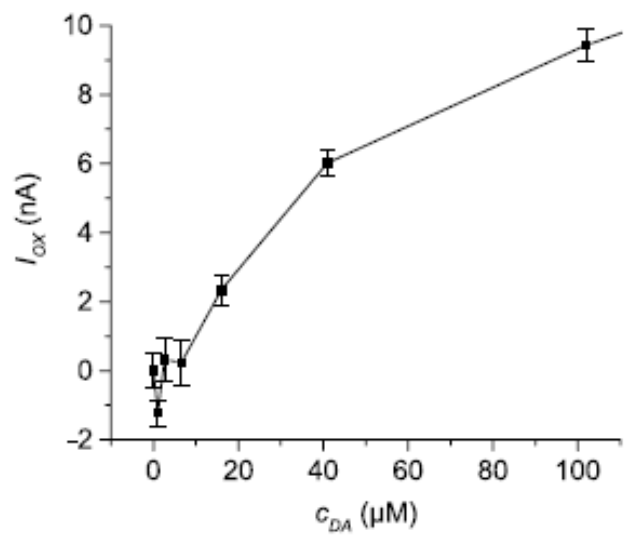


Fig. 4. Selectivity with respect to ascorbic acid. Background subtracted voltamogramms (forward scan) for samples with $200 \mu \mathrm{M}$ ascorbic acid and varying dopamine concentrations and a sample with $400 \mu \mathrm{M}$ ascorbic acid. The additional $200 \mu \mathrm{M}$ ascorbic acid have similar amplitude at $0.485 \mathrm{~V}$ as the $16 \mu \mathrm{M}$ dopamine sample.

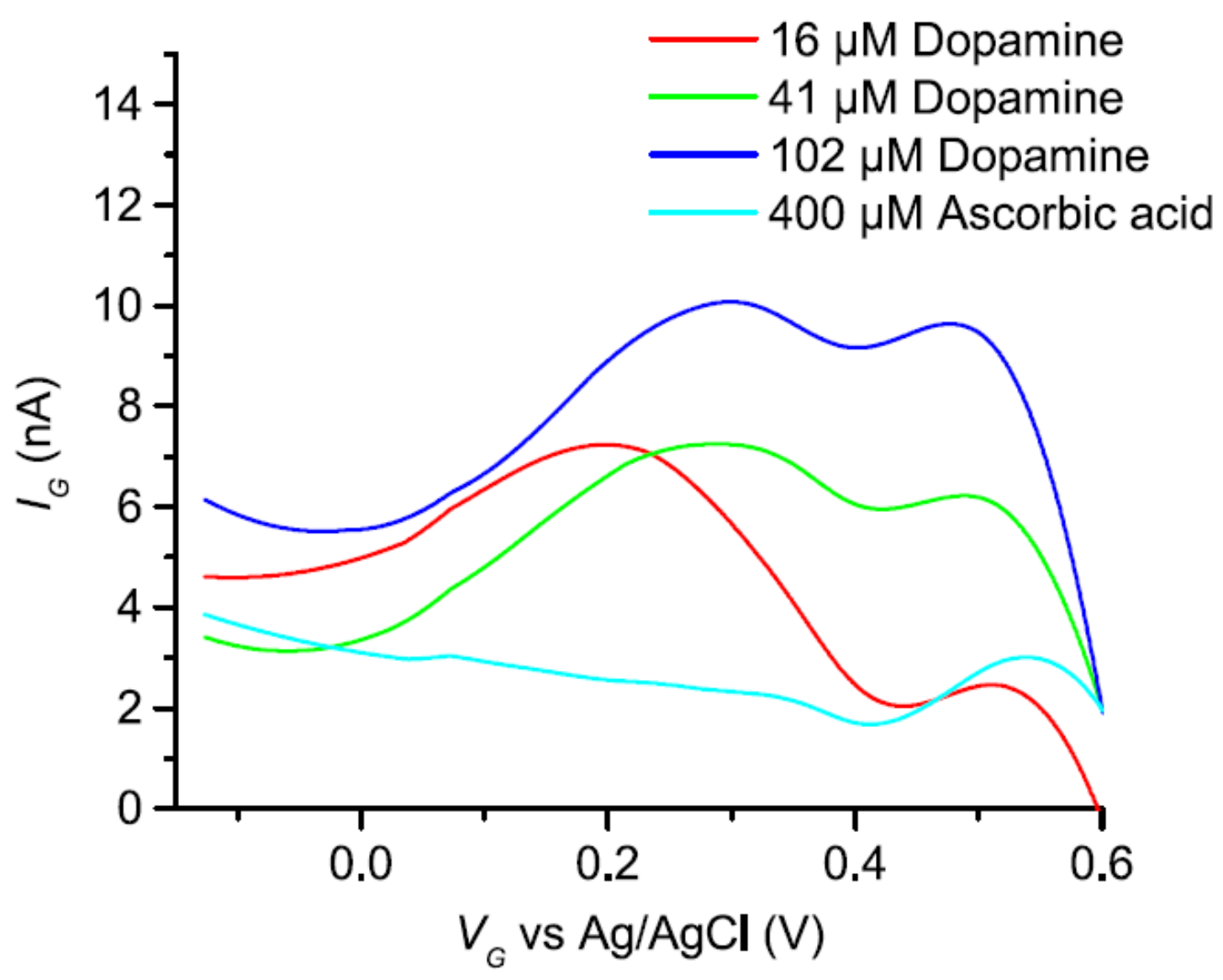


Fig. 5. Transient measurement of dopamine. (a) Color plot of background subtracted voltamogramms (forward scans) for a $10 \mathrm{~s}$ measurement in which dopamine was added at $\sim 3 \mathrm{~s}$ to a final concentration of $56 \mu \mathrm{M}$. The oxidation peak appears in yellow with the maximum at $0.5 \mathrm{~V}$. (b) The dopamine concentration obtained from the color plot at $0.485 \mathrm{~V}$. The current is converted into dopamine concentration by a linear fit in Figure $3 \mathrm{~d}$ for the lower concentrations.

(a)

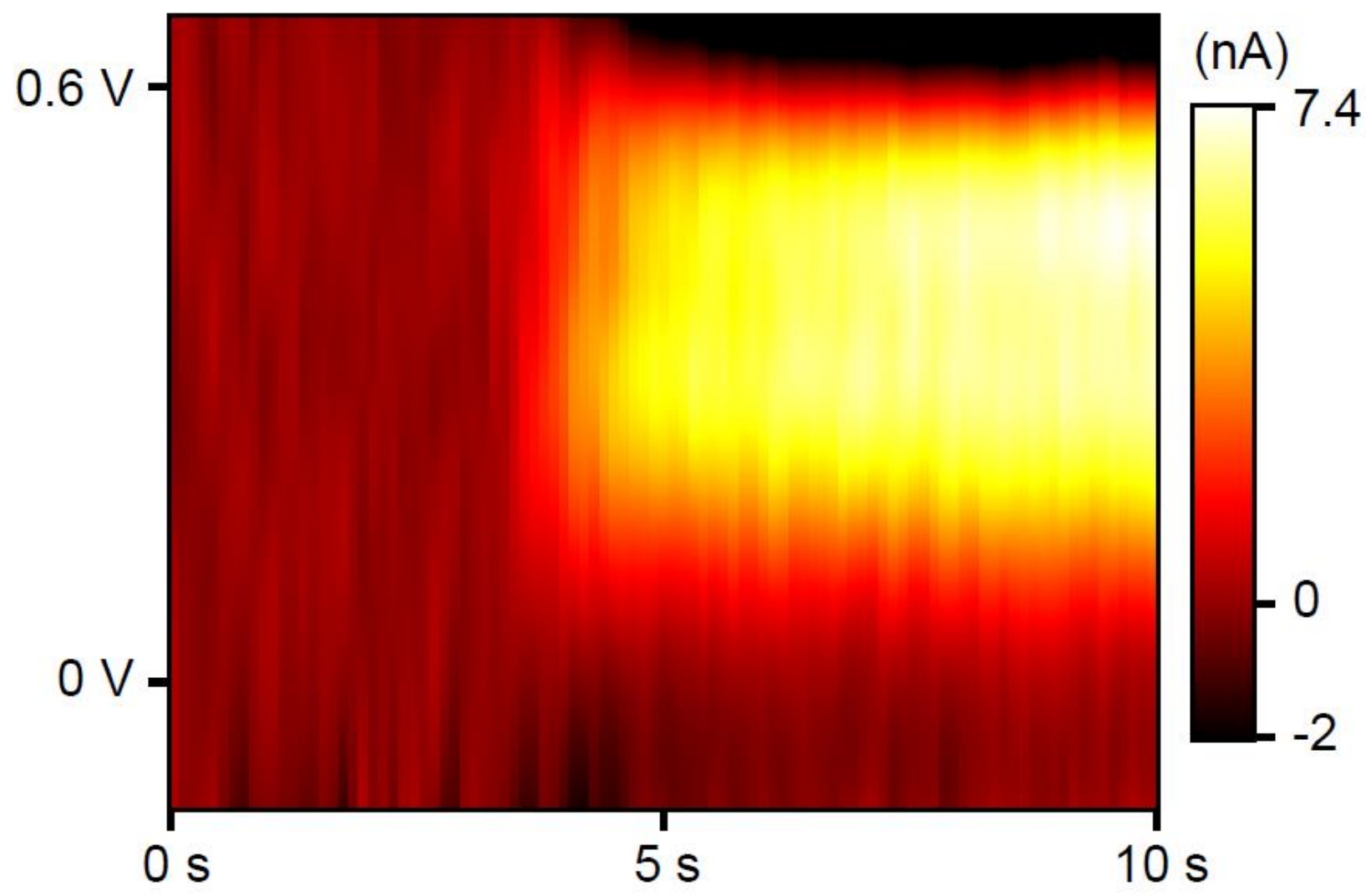




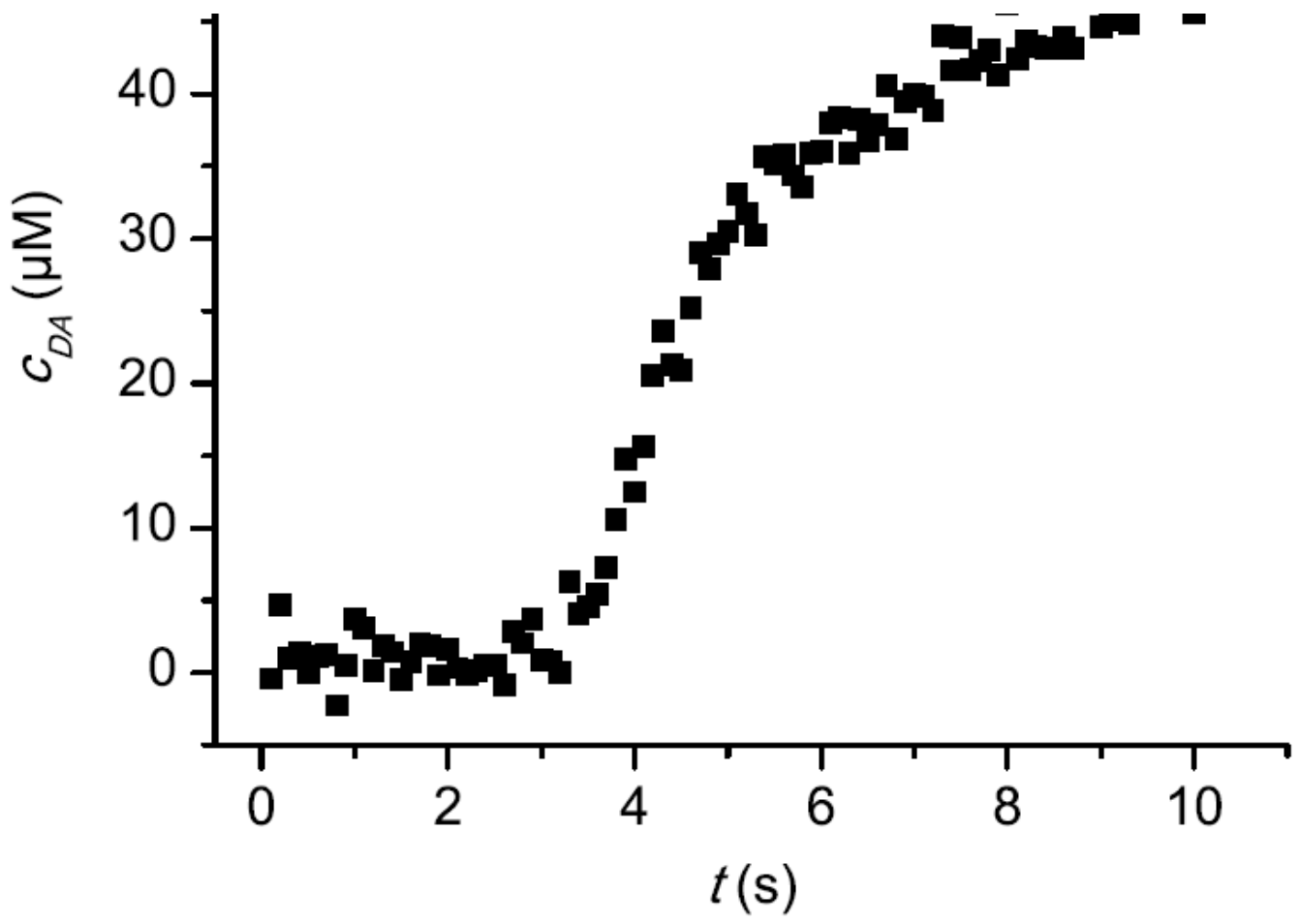




\section{References}

[1] A.C. Michael, L.M. Borland, Electrochemical Methods for Neuroscience, CRC Press, Boca Raton (FL), 2007.

[2] E.W. Crick, I. Osorio, N.C. Bhavaraju, T.H. Linz, C.E. Lunte, An investigation into the pharmacokinetics of 3-mercaptopropionic acid and development of a steady-state chemical seizure model using in vivo microdialysis and electrophysiological monitoring, Epilepsy Research, 74 (2007) 116-125.

[3] G.V. Rebec, J.R.C. Christensen, C. Guerra, M.T. Bardo, Regional and temporal differences in realtime dopamine efflux in the nucleus accumbens during free-choice novelty, Brain Res., 776 (1997) 6167.

[4] M. Heien, P.E.M. Phillips, G.D. Stuber, A.T. Seipel, R.M. Wightman, Overoxidation of carbon-fiber microelectrodes enhances dopamine adsorption and increases sensitivity, Analyst, 128 (2003) 14131419.

[5] M. Heien, A.S. Khan, J.L. Ariansen, J.F. Cheer, P.E.M. Phillips, K.M. Wassum, R.M. Wightman, Realtime measurement of dopamine fluctuations after cocaine in the brain of behaving rats, Proc. Natl. Acad. Sci. U. S. A., 102 (2005) 10023-10028.

[6] H. Sirringhaus, Device physics of Solution-processed organic field-effect transistors, Adv. Mater., 17 (2005) 2411-2425.

[7] J. Janata, M. Josowicz, Conducting polymers in electronic chemical sensors, Nat. Mater., 2 (2003) 19-24.

[8] F. Garnier, R. Hajlaoui, A. Yassar, P. Srivastava, ALL-POLYMER FIELD-EFFECT TRANSISTOR REALIZED BY PRINTING TECHNIQUES, Science, 265 (1994) 1684-1686.

[9] G.H. Gelinck, H.E.A. Huitema, E. Van Veenendaal, E. Cantatore, L. Schrijnemakers, J. Van der Putten, T.C.T. Geuns, M. Beenhakkers, J.B. Giesbers, B.H. Huisman, E.J. Meijer, E.M. Benito, F.J. Touwslager, A.W. Marsman, B.J.E. Van Rens, D.M. De Leeuw, Flexible active-matrix displays and shift registers based on solution-processed organic transistors, Nat. Mater., 3 (2004) 106-110.

[10] M. Hamedi, R. Forchheimer, O. Inganas, Towards woven logic from organic electronic fibres, Nat. Mater., 6 (2007) 357-362.

[11] D.A. Bernards, D.J. Macaya, M. Nikolou, J.A. DeFranco, S. Takamatsu, G.G. Malliaras, Enzymatic sensing with organic electrochemical transistors, J. Mater. Chem., 18 (2008) 116-120.

[12] D. Nilsson, M.X. Chen, T. Kugler, T. Remonen, M. Armgarth, M. Berggren, Bi-stable and dynamic current modulation in electrochemical organic transistors, Adv. Mater., 14 (2002) 51-54.

[13] D. Khodagholy, T. Doublet, P. Quilichini, M. Gurfinkel, P. Leleux, A. Ghestem, E. Ismailova, T. Herve, S. Sanaur, C. Bernard, G.G. Malliaras, In vivo recordings of brain activity using organic transistors, Nat. Commun., 4 (2013).

[14] D.A. Bernards, G.G. Malliaras, Steady-state and transient behavior of organic electrochemical transistors, Adv. Funct. Mater., 17 (2007) 3538-3544. 
[15] F. Cicoira, M. Sessolo, O. Yaghmazadeh, J.A. DeFranco, S.Y. Yang, G.G. Malliaras, Influence of Device Geometry on Sensor Characteristics of Planar Organic Electrochemical Transistors, Adv. Mater., 22 (2010) 1012-+.

[16] P. Lin, X.T. Luo, I.M. Hsing, F. Yan, Organic Electrochemical Transistors Integrated in Flexible Microfluidic Systems and Used for Label-Free DNA Sensing, Adv. Mater., 23 (2011) 4035-+.

[17] M. Kanungo, D.N. Srivastava, A. Kumar, A.Q. Contractor, Conductimetric immunosensor based on poly( 3,4-ethylenedioxythiophene), Chemical Communications, (2002) 680-681.

[18] P. Lin, F. Yan, J.J. Yu, H.L.W. Chan, M. Yang, The Application of Organic Electrochemical Transistors in Cell-Based Biosensors, Adv. Mater., 22 (2010) 3655-+.

[19] M. Asplund, T. Nyberg, O. Inganas, Electroactive polymers for neural interfaces, Polym. Chem., 1 (2010) 1374-1391.

[20] S.Y. Yang, B.N. Kim, A.A. Zakhidov, P.G. Taylor, J.K. Lee, C.K. Ober, M. Lindau, G.G. Malliaras, Detection of Transmitter Release from Single Living Cells Using Conducting Polymer Microelectrodes, Adv. Mater., 23 (2011) H184-H188.

[21] M.K. Zachek, A. Hermans, R.M. Wightman, G.S. McCarty, Electrochemical dopamine detection: Comparing gold and carbon fiber microelectrodes using background subtracted fast scan cyclic voltammetry, J. Electroanal. Chem., 614 (2008) 113-120.

\section{Biographies}

Klas Tybrandt received his MSc in Applied Physics and Electrical Engineering in 2007 and his PhD in Organic Electronics in 2012, both degrees from Linköping University. He is currently a Postdoctoral Fellow at the Laboratory of Organic Electronics, Department of Science and Technology, Linköping University. In 2013 he received an International Postdoc fellowship from the Swedish Research Council with start in 2014. His current research interests are organic bioelectronics and ionic circuits.

Suresh Babu Kollipara received his MSc in Physics in 2012 from Linköping University. He received his BEng in Electronics and Communication Engineering in 2007 from Andhra University, India. In 2012 he carried out his Master thesis work at the Laboratory of Organic Electronics, Department of Science and Technology, Linköping University.

Magnus Berggren received his MSc in Physics in 1991 and his PhD in 1996, both degrees from Linköping University. In 1997 he became the managing director of Thin Film Electronics AB. He then returned to Linköping University and also to a part time position at Swedish ICT-Acreo. Since 2002, he is the professor in Organic Electronics and is today also the director for the Laboratory for Organic Electronics, 
which includes 35 members. In 2006 he became the director of the Strategic Research Centre for Organic Bioelectronics and in 2011 he was elected a member of the Royal Swedish Academy of Sciences. 